\title{
Contrer les variants plus virulents du SRAS-CoV-2 demandera une réponse plus ingénieuse à la pandémie
}

\author{
Kirsten Patrick MD MB MSc
}

Citation : CMAJ 2021 October 25;193:E1633-4. doi : 10.1503/cmaj.211656-f; diffusion hâtive le 5 octobre 2021

Voir la version anglaise de l'article ici : www.cmaj.ca/lookup/doi/10.1503/cmaj.211656; voir l'article connexe en anglais ici : www.cmaj. ca/lookup/doi/10.1503/cmaj.211248

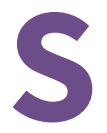

elon de récentes recherches, les variants sous surveillance rehaussée (VSSR) du SRAS-CoV-2 sont plus virulents que la souche initiale, et plus contagieux $x^{1}$. Fisman et Tuite ont utilisé les données sur les cas de COVID-19 en Ontario pour estimer la virulence des variants Alpha/B1.1.17, Beta/B.1.351, Gamma/P.1 et Delta/B.1.617 comparativement à celle de la souche de type sauvage initiale du SRAS-CoV-2. Ils ont constaté des risques plus élevés d'hospitalisation, d'admission aux unités de soins intensifs et de décès avec les VSSR, particulièrement le variant Delta.

Le Canada fait actuellement face à une pandémie différente de celle qu'il a affrontée au début de 2020. Le virus est devenu plus pernicieux et plus menaçant, ce qui signifie que nous devons aussi nous montrer plus ingénieux. Au Canada, les gouvernements peuvent préserver la sécurité de la population en appliquant des politiques qui combinent intelligemment toutes les mesures avérées efficaces.

L'arrivée hâtive des vaccins contre le SRAS-CoV-2 a suscité l'espoir que les consignes puissent être levées et que la vie puisse reprendre son cours normal plus rapidement que prévu. Les gens sont bien sûr fatigués des confinements successifs, des mesures de distanciation physique et des restrictions imposées aux commerces; l'incidence économique et psychologique des restrictions dues à la pandémie a été immense. À l'approche de l'été, certains gouvernements ont choisi de mettre sur " pause » la plupart des mesures de santé publique. En Alberta et en Saskatchewan, par exemple, les responsables ont décidé de faire fin des mises en garde des scientifiques au sujet de la menace des variants sous surveillance rehaussée; à la place, ils ont prôné l'espoir en faisant miroiter «le meilleur des étés $»^{2}$. Aujourd'hui, leurs systèmes de santé, en particulier les unités de soins intensifs, sont sollicités comme jamais auparavant; les travailleurs de la santé sont épuisés, accablés moralement, et l'effet domino des interventions annulées affectera la santé de la population pendant des années encore.
La plus grande virulence des variants du SRAS-CoV- $2^{1}$, leur capacité de causer des éclosions chez des personnes entièrement vaccinées ${ }^{3}$ et certains rapports faisant état d'une efficacité moindre des vaccins approuvés contre le variant Delta ne sont pas les seuls facteurs à tamiser la fameuse lumière au bout du tunnel pandémique. Il faut aussi déplorer une malheureuse pandémie de désinformation qui entraîne la réticence et le refus du vaccin à grande échelle au Canada et ailleurs ${ }^{4,5}$; cela met en péril les efforts pour atteindre l'immunité collective contre le SRAS-CoV-2 qui permettrait d'alléger les mesures de santé publique ${ }^{6}$.

Les vaccins contre le SRAS-CoV-2 préviennent efficacement les cas graves de COVID-19 et les décès; selon les données nordaméricaines, la plupart des patients atteints de COVID-19 qui ont actuellement besoin de soins intensifs et de soutien respiratoire sont non vaccinés ${ }^{7,8}$. Donc, chaque Canadien et Canadienne admissible chez qui le vaccin n'est pas contre-indiqué devrait le recevoir pour protéger les enfants de moins de 12 ans qui n'ont pas encore accès au vaccin et les personnes exposées à des facteurs de risque de COVID sous sa forme grave. Les gouvernements devraient intensifier leurs efforts pour offrir les vaccins aux personnes admissibles et prioriser l'approbation de vaccins sécuritaires pour les enfants.

La vaccination obligatoire et le passeport vaccinal sont des initiatives qui ont persuadé beaucoup de personnes jusqu'alors réticentes à recevoir le vaccin. Même si leur nature obligatoire a pu soulever de navrantes protestations, l'obligation de la vaccination pour travailler ou accéder à certains milieux est justifiée; la Commission des droits de la personne de l'Ontario a récemment statué sur le fait que les personnes qui entretiennent des croyances antivaccin ou antimasque ont le droit de ne pas utiliser ces protections, mais n'ont pas le droit de menacer la santé d'autrui $^{4}$. Les gouvernements devraient élargir la vaccination obligatoire et exiger que plus de travailleurs des secteurs publics qui œuvrent auprès de groupes, comme les professeurs, priorisent avant tout la sécurité dans l'exercice de leurs fonctions. 
Les manifestations sont moralement injustifiées et ne devraient pas être autorisées là où elles risquent de menacer le bien-être d'autrui ou de nuire à la prestation de services essentiels. Les gouvernements peuvent interdire aux manifestants de protester à proximité des écoles et des hôpitaux; l'Assemblée nationale du Québec a récemment adopté une loi qui interdit aux antivaccins de manifester près des écoles, des garderies, des hôpitaux, des cliniques de dépistage et de vaccination pour la COVID-19 ${ }^{9}$. Les travailleurs de la santé et autres travailleurs essentiels au Canada ont le droit de se sentir en sécurité et respectés lorsqu'ils se présentent au travail; ce sont eux qui permettent à la société de continuer de bien fonctionner.

Les gouvernements devraient aussi offrir une aide financière et technique aux écoles pour rendre les environnements plus sécuritaires et améliorer la ventilation dans les classes. Le déploiement des tests antigéniques rapides pour les personnes asymptomatiques ${ }^{10}$ et l'optimisation de la recherche de contacts dans les cas d'éclosion permettront de protéger les enfants qui ne peuvent pas encore être vaccinés et de réduire la circulation du SRAS-CoV-2 dans les collectivités.

Identifier rapidement les éclosions et s'assurer que les personnes infectées par le SRAS-CoV-2 se placent en isolement demeure une importante partie de notre défense contre la pandémie et contre un variant du SRAS-CoV-2 de plus en plus virulent. La façon la plus rapide d'empêcher tout variant du SRAS-CoV-2 de se propager est que moins de personnes respirent le même air ${ }^{11}$. Cela signifie que tous les ordres de gouvernements doivent répondre adroitement et avec fermeté aux éclosions émergentes en réactivant temporairement les mesures de distanciation physique appuyées par un message clair.

En terminant, il est impératif que le Canada appuie les efforts pour accélérer la vaccination dans les pays où cette dernière laisse à désirer en faisant don de ses stocks excédentaires et en entérinant les accords mondiaux qui permettront aux pays de fabriquer leurs vaccins localement. Les voyages internationaux ont repris, et avec la menace permanente qu'ils représentent quant à l'émergence et la propagation de nouveaux variants sous surveillance rehaussée de la COVID-19 en l'absence d'une réponse globale coordonnée, ils projetteront une ombre inquiétante sur nos vies pendant des années encore.

Alors que le Canada s'apprête à vivre un deuxième hiver de pandémie de COVID-19, nous devons collectivement apprendre des erreurs passées et nous assurer d'utiliser tous les outils à notre disposition pour éviter de prochains confinements et empêcher une dégradation plus marquée de notre infrastructure sanitaire.

\section{Références}

1. Fisman DN, Tuite AR. Evaluation of the relative virulence of novel SARS-CoV-2 variants: a retrospective cohort study in Ontario, Canada. CMAJ 2021 October 5 [cyberpublication avant impression]. doi: $10.1503 / \mathrm{cmaj} .211248$.

2. Austen I. Alberta's 'best summer ever' ends with an overwhelmed medical system. The New York Times 2021 Sept. 24. Accessible ici : https://www.nytimes. com/2021/09/24/world/canada/canada-alberta-covid-cases.html (consulté le 25 sept. 2021).

3. Bergwerk M, Gonen T, Lustig Y, et al. COVID-19 breakthrough infections in vaccinated health care workers. N Engl J Med 2021 July 28 [cyberpublication avant impression]. doi: 10.1056/NEJMoa2109072.

4. OHRC policy statement on COVID-19 vaccine mandates and proof of vaccine certificates. Toronto: Ontario Human Rights Commission; 2021. Accessible ici : www. ohrc.on.ca/en/news_centre/ohrc-policy-statement-covid-19-vaccine-mandates -and-proof-vaccine-certificates (consulté le 25 sept. 2021).

5. Anti-vaccine protesters clash with police In Melbourne, Australia, for the 2nd day. NPR (National Public Radio) 2021 Sept. 21. Accessible ici : https://www.npr.org /2021/09/21/1039301977/anti-vaccine-protesters-clash-with-police-in-melbourne -for-the-second-straight-d (consulté le 25 sept. 2021).

6. Bolotin S, Wilson S, Murti M. Achieving and sustaining herd immunity to SARSCoV-2. CMAJ 2021;193:E1089.

7. Confirmed cases of COVID-19 following vaccination in Ontario: December 14, 2020 to September 4, 2021 [communiqué de presse]. Toronto: Public Health Ontario; 2021 Sept. 4. Accessible ici : https://www.publichealthontario.ca/-/ media/documents/ncov/epi/covid-19-epi-confirmed-cases-post-vaccination. pdf?la=en (consulté le 25 sept. 2021).

8. Scobie HM, Johnson AG, Suthar AB, et al. Monitoring incidence of COVID-19 cases, hospitalizations, and deaths, by vaccination status: 13 U.S. jurisdictions, April 4-July 17, 2021. MMWR Morb Mortal Wkly Rep 2021;70:1284-90.

9. Ross S, Lofaro J. Quebec swiftly passes new bill to prohibit anti-vaccine protests near schools, hospitals. CTV News Montreal 2021 Sept. 23, updated 2021 Sept. 24. Accessible ici : https://montreal.ctvnews.ca/quebec-swiftly-passes-new-bill-to -prohibit-anti-vaccine-protests-near-schools-hospitals-1.5597380 (consulté le 25 sept. 2021).

10. Schwartz KL, McGeer AJ, Bogoch II. Rapid antigen screening of asymptomatic people as a public health tool to combat COVID-19. CMAJ 2021;193:E449-52.

11. Addleman S, Leung V, Asadi L, et al. Mitigating airborne transmission of SARSCoV-2. CMAJ 2021;193:E1010-1.

Intérêts concurrents : Voir www.cmaj.ca/site/misc/cmaj_staff.xhtml.

Affiliation : Rédactrice en chef [par intérim], JAMC

Propriété intellectuelle du contenu : Il s'agit d'un article en libre accès distribué conformément aux modalités de la licence Creative Commons Attributions (CC BY-NC-ND 4.0), qui permet l'utilisation, la diffusion et la reproduction dans tout médium à la condition que la publication originale soit adéquatement citée, que l'utilisation se fasse à des fins non commerciales (c.-à-d., recherche ou éducation) et qu'aucune modification ni adaptation n'y soit apportée. Voir : https://creativecommons.org/licenses/by-nc-nd/4.0/deed.fr.

Correspondance : Rédaction du CMAJ, editorial@cmaj.ca 